\title{
Risk assessment: workers operating in loading/unloading (shipping/receiving) areas
}

\author{
E. Salvador \& M. Forte \\ Axa Matrix Risk Consultants, Italy
}

\begin{abstract}
Loading/unloading areas are essential for many businesses, including production facilities, logistic centres, product receiving/shipping centres, department stores etc. These areas can be very dangerous for workers due to the type of operation, the use of mobile equipment and the frequent interferences between workers and mobile equipment.

This presentation is a quantitative methodology (isorisk maps) to analyse and map the risks related to loading/unloading areas with the aim of comparing the various levels of exposure for workers, considering different technical and design solutions and with respect to applicable procedures.

This methodology is based on the identification of a risk assessment criteria (risk grading) from which it is possible to extract quantitative indications of the vulnerability level (probability), severity (loss potential) for each site and the impact of single risk factors.
\end{abstract}

Keywords: risk assessment, vulnerability, severity, loading/unloading areas, shipping/receiving areas, isorisk maps.

\section{Isorisk maps}

As already described in Forte and Salvador [1] this methodology for identifying and representing the risk is based on the evaluation of vulnerability and severity for each identified scenario. Vulnerability is defined as a measure of the frequency and probability in normal operative conditions. Severity is defined as the quantification of loss expectancy in normal operative conditions with prevention and protection systems.

Using these criteria, it is possible to produce graphs vulnerability-severity (so-called "isorisk maps"), to graphically represent the exposure to a given peril. 
The graph refers to the traditional risk definition: $R=S \cdot V$, where $\mathrm{S}$ is the severity, $\mathrm{V}$ the vulnerability and $\mathrm{R}$ the risk.

As it is not possible to define a "mathematical" value of the frequency, an alternative value, defined as "vulnerability", is used. This vulnerability is based on the evaluation and subsequent elaboration of some typical indicators of the event frequency.

In the graph, each location (a production facility, a warehouse or any other type of facility) is represented with a dot whose position is defined by the vulnerability and severity values (defined in the following paragraphs). Figure 1 is an example.

This methodology will be applied to assess the risk for operators working in loading/unloading areas.

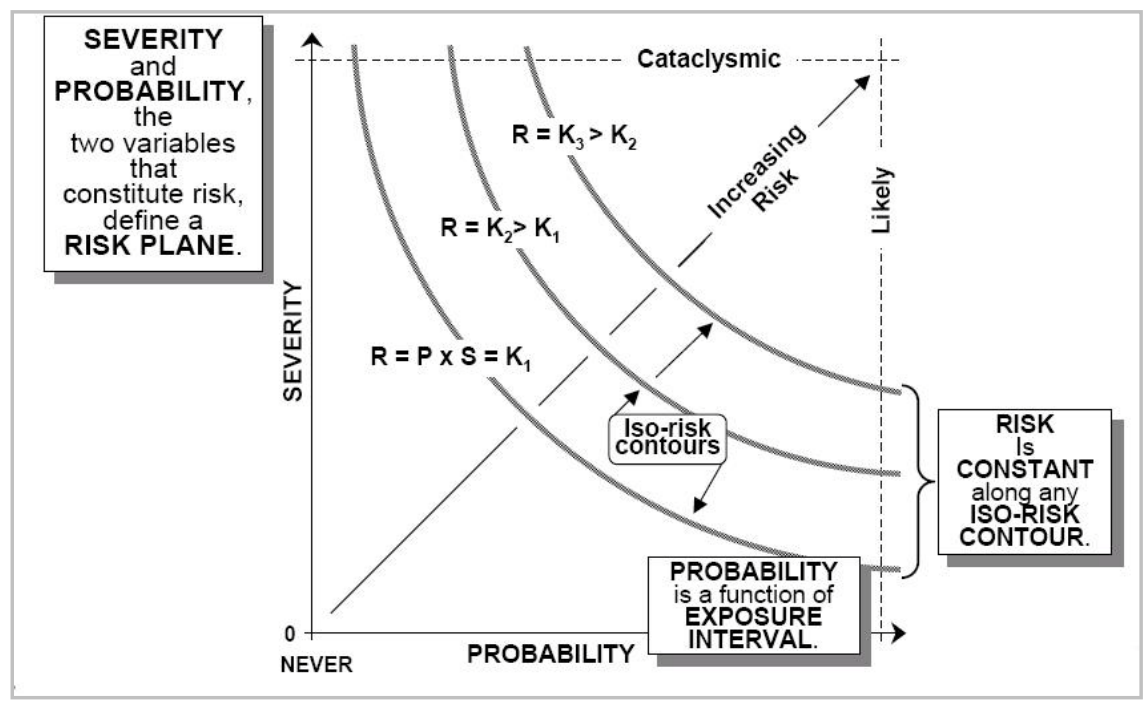

Figure 1: $\quad$ Isorisk map.

\section{Loading/unloading docking bays}

This paper refers to loading docks in which the connection between the floor and the truck is made through electro-hydraulic ramps. This connection must be correctly positioned to guarantee stability during the passage of mobile equipment or operators used to load or unload the truck.

Typically, loading docks are at least $1.20 \mathrm{~m}$ high and are as long as needed to cover the front of the service area to protect equipment operations from weather (as shown in Figure 2). 


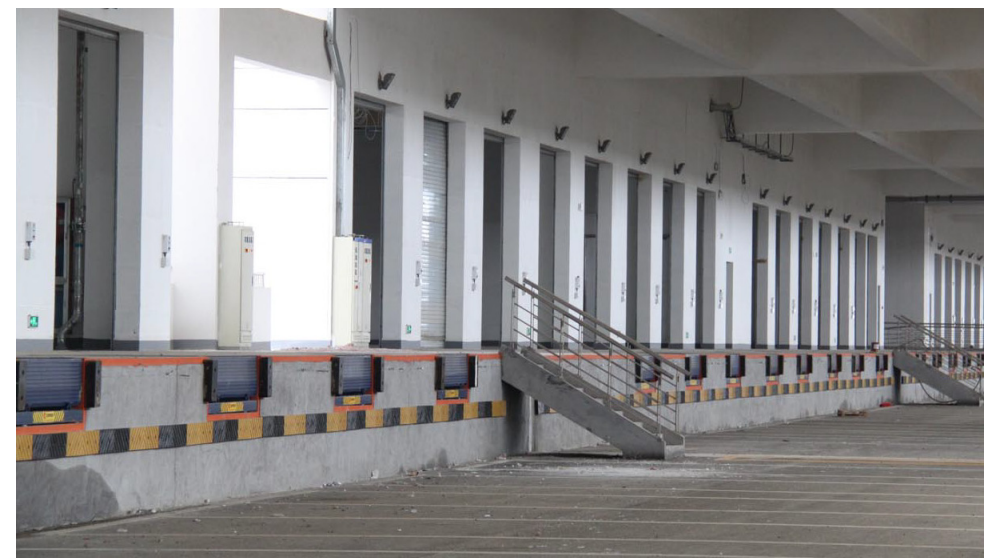

Figure 2: $\quad$ Loading/unloading docks.

Loading ramps and docking bays have specific characteristics and are referenced in the Health and Safety rules in work areas (see references).

These work areas are often identified as the most dangerous in many Risk Assessment documents, and are therefore subject to particular attention by Prevention and Protection Officers. Evaluations are usually based on qualitative or semi-quantitative criteria, using a risk matrix where higher values correspond to higher risks. However, an in-depth analysis of the areas is usually lacking, thus preventing the identification of the main risk factors on which to intervene with adequate prevention and protection measures.

Assuming typical work rules are respected, the following methodology provides a tool to compare different physical and human arrangements to assess the aspects most affecting a loading/unloading area risk.

\section{Methodology to develop a grading system for quantifying the risk}

The creation of an evaluation system (grading) of the risk in loading/unloading areas (ref. Figure 3 ) is based on a series of activities, summarised as follows:

- Breakdown of loading and unloading area into macroareas

- Analysis of the main risks present in the macroareas

- "Engineered" analysis of the macroareas and definition of a set of variables

- Definition of algorithms for risk quantification

This grading is based on the definition of a set of variables, each one associated with two or more "closed" answers corresponding to ratings used by algorithms for risk quantification.

This data input structure allows the distribution of the grading via a checklist sent to various locations. Collected information is then converted into numerical indications through a system of weights and algorithms, guaranteeing the objectiveness of the analysis. 


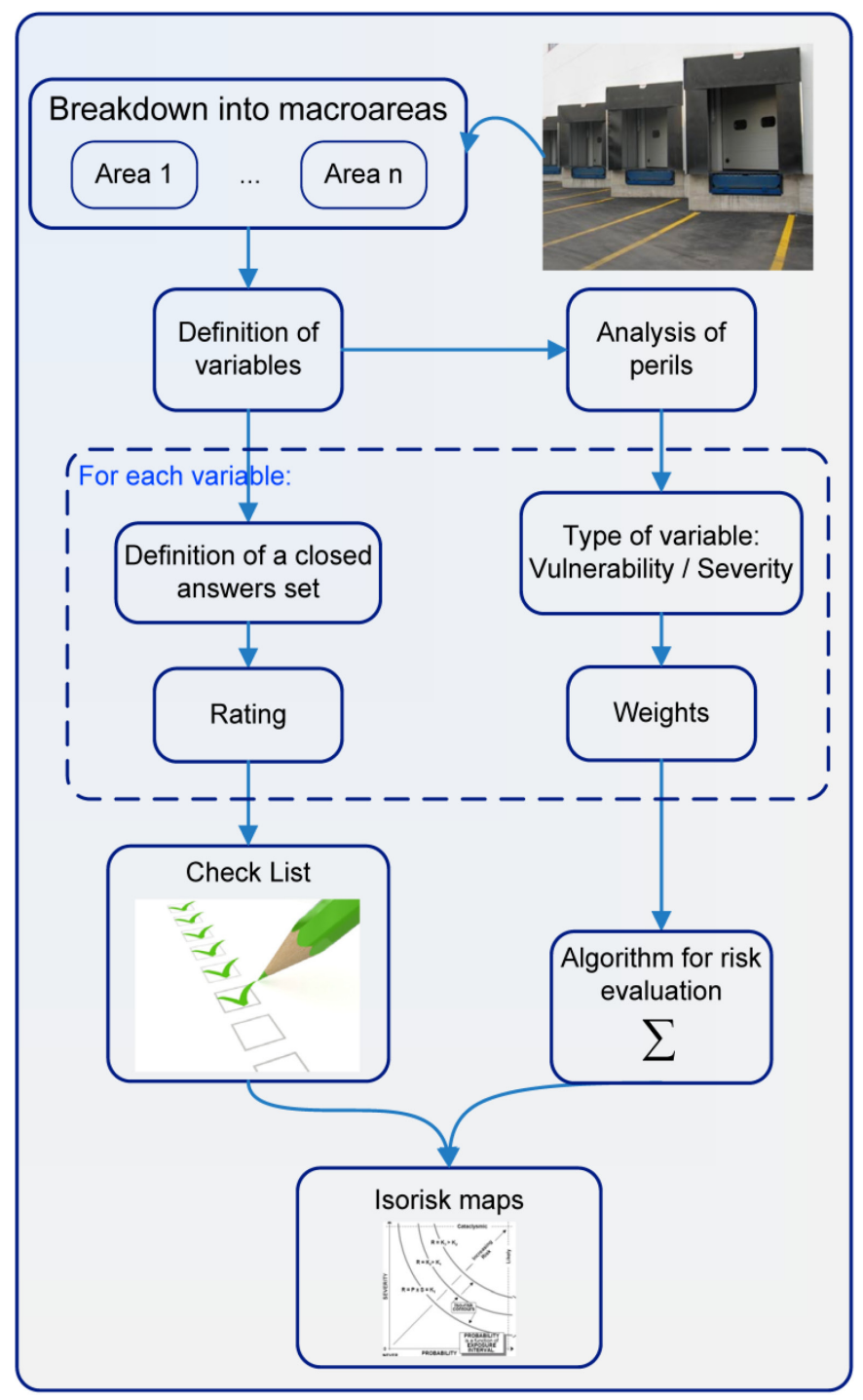

Figure 3: Analysis methodology.

\subsection{Breakdown of loading/unloading areas into macroareas}

The analysis of areas used for product loading/unloading operations through docking bays equipped with motorized ramps identifies six possible macroareas, each with features allowing the development and the creation of a set of dedicated variables.

The identified macroareas can be further subdivided into physical areas (physical aspects) and human element aspects, as shown in Figure 4. 


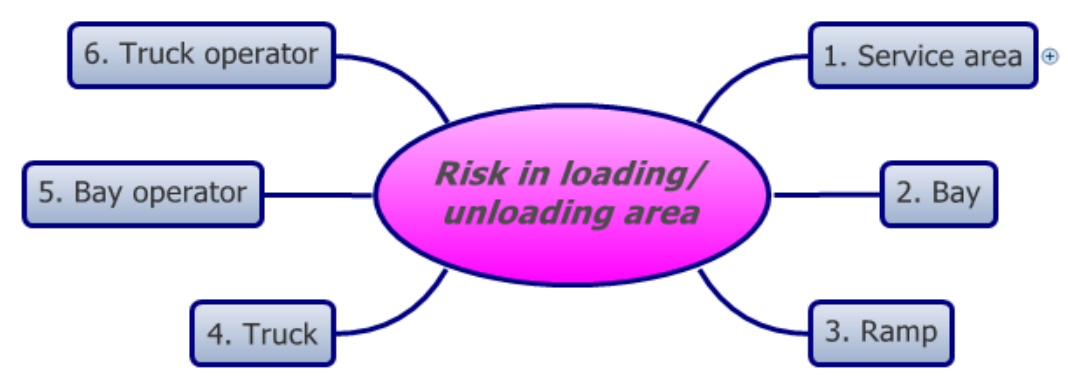

Figure 4: Breakdown into macroareas.

Physical elements

1. Service area area in the front of the docking bay, dedicated to trucks moving

2. Docking Bay area raised with respect to the service area, dedicated to the ramp and docking facilities and to the mobile or manual means to manoeuvre goods from the truck.

3. Ramp

4. Truck hooked or telescopic, used to connect the docking bay and the truck

truck must approach the docking bay and be docked for loading/unloading operations.

Human Element aspects

5. Bay operator

responsible for loading and unloading operations from the truck.

6. Truck operator truck driver (employee of the Customer or third company).

Each area has been analysed to highlight the variables and the perils affecting the associated risk.

\subsection{Main perils present in the macroareas}

Considering the identified macroareas and the kinds of activities present, the majority of perils can be linked to:

- Fall

Falls are possible from the bay (usually placed at 1,5-2 $\mathrm{m}$ higher with respect to the service area) due to an early departure of the truck or to inadequate anti-fall protection means on the docking bay.

- $\quad$ Trip/Slippage

Trip peril can be due to the presence of protrusions on the floor (ex. the ramp is lifted from the bay, or presence of truck guiding tracks in the service 
area); slippage can be due to slippery surfaces (ex. for macroclimatic aspects not adequately managed).

- Accident

In the service and docking areas, due to the presence of vehicles and operators in movement.

- $\quad$ Crush/hit

Crushing of a worker by a moving vehicle against a wall or pallet load, or a hit between the vehicle and a worker operating in the service or docking areas.

\subsection{Analysis of the areas and creation of a set of variables}

The analysis then further defines a wide number of variables linked to the various macroareas. As per the example in Figure 5 below, these variables are defined for the macroarea: "Service Area".

A weight is then attributed to each variable, based on a predetermined criteria for each of the perils it is connected to.

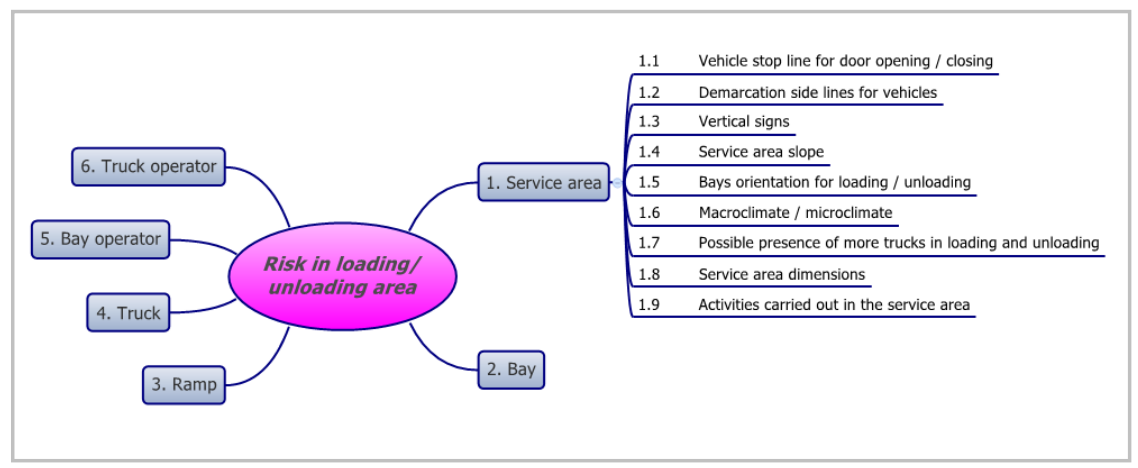

Figure 5: Details of variables for the service area.

The analysis process, then weights each variable as a function of each connected peril, and the effect in terms of vulnerability and/or severity. This is outlined in the following table, showing the analysis done for the presence of lateral demarcation lines of trucks in the service area.

\section{Risk quantification}

To allow a self-assessment, a set of "closed" answers is assigned to each question.

The answers are then modified by an algorithm which associates them to a rating. Together with the previously described weights, this quantifies the contribution of a single element to the vulnerability and severity. 
Table 1: $\quad$ Example of a checklist, relative to variable 1.2 of Figure 4.

\begin{tabular}{|c|c|c|c|}
\hline \multicolumn{3}{|l|}{ Area: } & \\
\hline 1. & \multicolumn{2}{|c|}{ Service area } & \\
\hline \multicolumn{3}{|l|}{ Variable: } & \\
\hline 1.2 & \multicolumn{2}{|c|}{$\begin{array}{l}\text { Truck lateral } \\
\text { demarcation lines }\end{array}$} & \\
\hline \multicolumn{4}{|c|}{ Type of variable (vulnerability/severity): } \\
\hline $\mathbf{V}$ & \multicolumn{3}{|c|}{$\begin{array}{l}\text { This element affects only the vulnerability, as it does not } \\
\text { represent a physical obstacle to the occurrence of a loss, but } \\
\text { it can decrease its probability. }\end{array}$} \\
\hline \multicolumn{4}{|l|}{ Weights: } \\
\hline \multicolumn{2}{|l|}{ Fall } & 0 & $\begin{array}{l}\text { Not applicable in the service area as there is no } \\
\text { height difference: null. }\end{array}$ \\
\hline \multicolumn{2}{|c|}{ Stumble/Slippage } & 0 & $\begin{array}{l}\text { The presence/lack of lateral demarcation lines } \\
\text { does not affect the possibility of } \\
\text { tripping/slippage of the workers: null }\end{array}$ \\
\hline \multicolumn{2}{|l|}{ Accident } & $\mathrm{W}_{3}$ & \multirow{2}{*}{$\begin{array}{l}\text { The presence of demarcation lines in the service } \\
\text { area helps the drivers while approaching the } \\
\text { truck to the docking bay, decreasing the } \\
\text { possibility (vulnerability) of an accident/hit of } \\
\text { the workers: weight not null, to be defined } \\
\text { according to the quantification criteria adopted. }\end{array}$} \\
\hline Crush/Hit & & $\mathrm{W}_{4}$ & \\
\hline
\end{tabular}

To obtain risk indicators useful to compare the areas between the various locations, these ratings are elaborated to obtain, respectively:

- Vulnerability $V_{p}$ and severity $S_{p}$ associated to each peril, irrespective of the area;

- Vulnerability $\mathrm{V}_{\mathrm{a}}$ and severity $\mathrm{S}_{\mathrm{a}}$ associated to each area, irrespective of the peril;

- Global vulnerability $\mathrm{V}_{\mathrm{g}}$ and severity $\mathrm{S}_{\mathrm{g}}$ associated with the loading/unloading area of the location, considering the contributions of all the variables in each area and associated with each peril.

The following is a description of the expressions used to evaluate $\mathrm{V}$ and $\mathrm{S}$ in the three mentioned cases. 
Table 2: $\quad$ Vulnerability and severity parameters.

\section{Parameters $V$ and $S$, divided per peril (contribution from all the variables)}

$$
V_{p}=\sqrt{\frac{\sum_{j=1}^{N}\left(\text { rating }_{j} \cdot w_{p, j}\right)^{2}}{N}}
$$

$$
S_{p}=\sqrt{\frac{\sum_{j=1}^{N}\left(\text { rating }_{j} \cdot w_{p, j}\right)^{2}}{N}}
$$

\begin{tabular}{|c|c|c|}
\hline \multirow{4}{*}{ where: } & $V_{p}$ & is the vulnerability associated to peril p-nth; \\
\hline & $S_{p}$ & is the severity associated to peril p-nth; \\
\hline & rating $_{j}$ & is the rating associated to variable $\mathrm{j}$-nth; \\
\hline & $w_{p, j}$ & $\begin{array}{l}\text { Represents the weight associated to variable j-nth } \\
\text { connected to peril i-nth (fall, stumble/slippage, } \\
\text { accident, crush/hit). }\end{array}$ \\
\hline
\end{tabular}

\section{Parameters $V$ and $S$ divided per area (contribution from all the variables} connected to a specific area)

\begin{tabular}{|c|c|c|}
\hline \multirow{5}{*}{ where: } & $V_{a}$ & is the vulnerability associated to area a; \\
\hline & $S_{a}$ & is the severity associated to area a; \\
\hline & rating $_{j}$ & is the rating associated to variable $\mathrm{j}$-nth; \\
\hline & $w_{i, j}$ & $\begin{array}{l}\text { Represents the weight associated to variable j-nth } \\
\text { connected to peril i-nth (fall, stumble/slippage, } \\
\text { accident, crush/hit); }\end{array}$ \\
\hline & $n$ & $\begin{array}{l}\text { Represents the number of variables influencing the } \\
\text { area under survey }\end{array}$ \\
\hline
\end{tabular}

$$
V_{a}=\frac{\sum_{i=1}^{4} \frac{\sum_{j=1}^{n}\left(\text { rating }_{j} \cdot w_{i, j}\right)^{2}}{n}}{4} \quad S_{a}=\frac{\sum_{i=1}^{4} \frac{\sum_{j=1}^{n}\left(\operatorname{rating}_{j} \cdot w_{i, j}\right)^{2}}{n}}{4}
$$


Table 2: $\quad$ Continued.

\section{Global parameters $V$ and $S$}

\begin{tabular}{|l|l|l|}
\hline & $V_{g}=\sqrt{\frac{\sum_{a=1}^{4} V_{a}^{2}}{4}} \quad S_{g}=\sqrt{\frac{\sum_{a=1}^{4} S_{a}^{2}}{4}}$ \\
\hline where: & $V_{a}$ & is the vulnerability associated to area a; \\
\hline & $S_{a}$ & is the severity associated to area a; \\
\hline
\end{tabular}

\subsection{Development of isorisk maps}

The resulting grading is used to compare the risk associated with the loading/unloading areas of the sites under analysis through isorisk maps (vulnerability-severity graphs).

To better evaluate all the aspects that impact on the risk of these areas, it is possible to create two maps:

- Isorisk - Peril

In this kind of graph, with coordinates vulnerability-severity, each kind of peril is represented with a dot, together with the dot representing the evaluation of the area under survey.

- Isorisk - Areas

Results obtained for the variables are then aggregated to get an evaluation of vulnerability and severity for each of the six previously mentioned areas.

In this way, each dot represents (in relation to all described perils) the contribution to global risk of the area.

Besides the dots indicating the risk associated to areas and perils, each graph contains a rectangle representing the extreme values (in terms of vulnerability and severity) which can be reached by the global risk of the loading/unloading area of the site. This reference is useful to evaluate the gap between the risk value calculated for the single area and the theoretical maximum and minimum values of the defined grading.

Taking as an example a generic Site whose analysis has given the following values:

- Vulnerability $=37.75$

- Severity $=46.50$

it is possible to obtain the representations shown in Figures 5 and 6.

In both graphs, a dot representing the risk value of the site in terms of vulnerability and severity is indicated. In the Figure 6 graph, the impact of the single areas on the global risk is shown, while in Figure 7 graph the contribution to risk given by the various perils is indicated. The combination of the two representations allows us to analyze in-depth which are the risk factors with a greater impact on the single perils present in the various areas. 


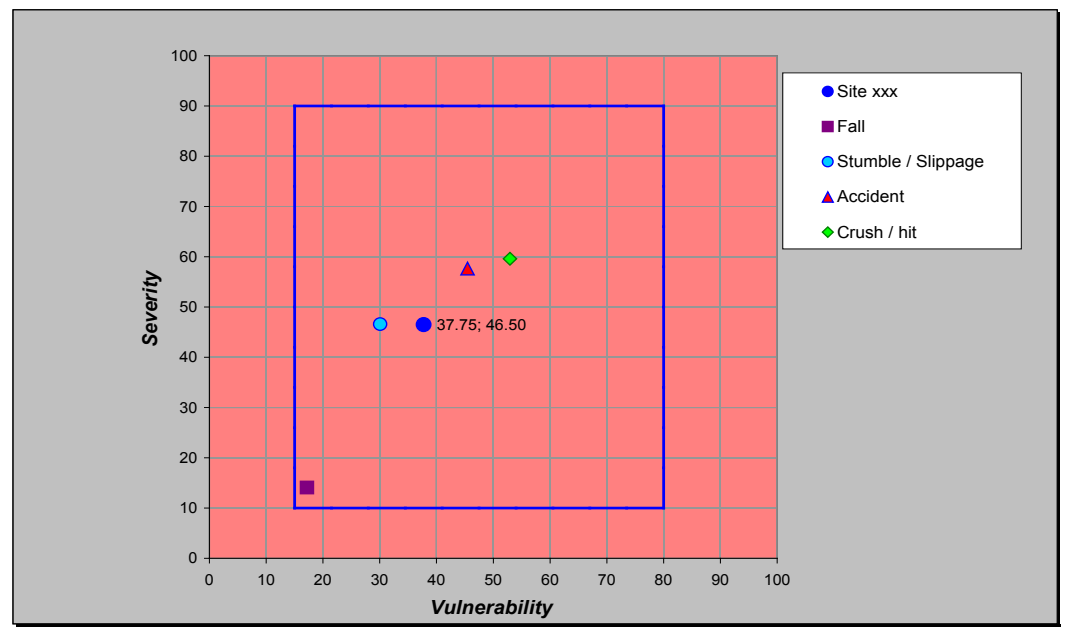

Figure 6: Macroareas contribution to risk.

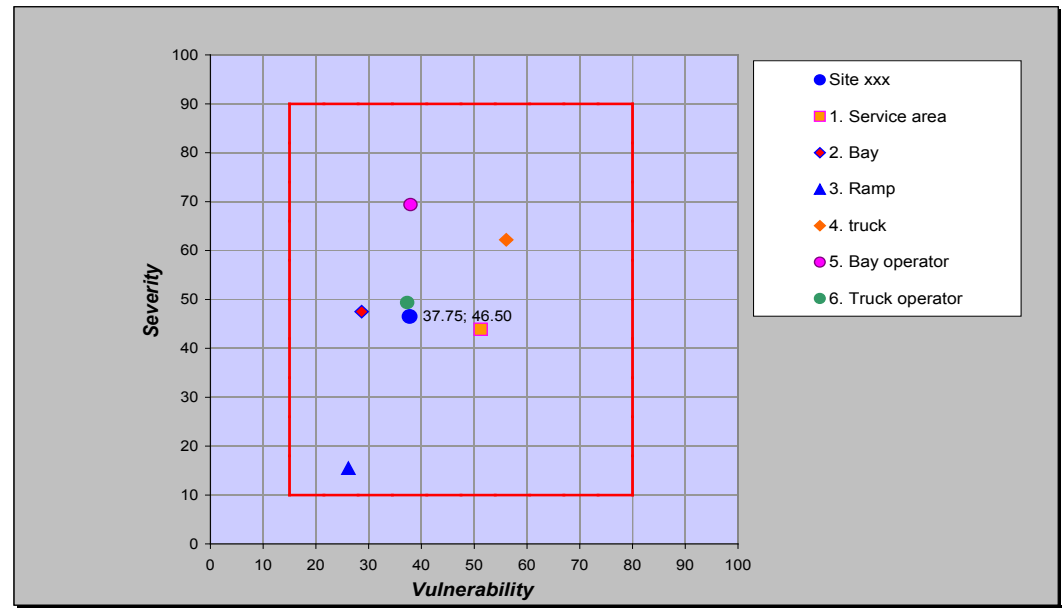

Figure 7: Perils contribution to risk.

In the previous figures, the vulnerability and severity evaluations for each site originate not only from the physical aspects but also from observation, during the visit, of the application of the procedures (human element) and type of trucks used.

To compare the various sites without any influence from these variables but taking into account only the physical elements, it is possible to prepare simulations for each site assigning the same rating to all the variables relating to 
these aspects (the lowest, corresponding to the solution with the smallest impact on the risk).

This isorisk, shown in Figure 8, is very useful for a Gap Analysis, aimed at measuring the gap between the risk assessment from the visit and the minimum risk attributable to each site. This underlines that this assessment tool can be effectively applied to monitor the risk for this kind of areas and to highlight any critical point to intervene on.

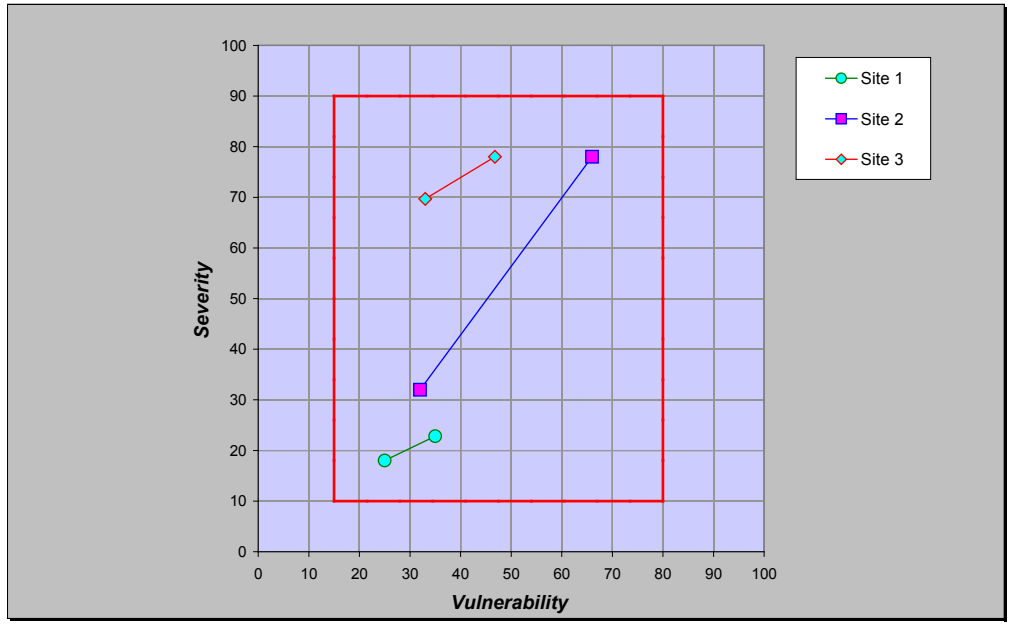

Figure 8: Benchmarking between a group of sites and gap analysis.

\section{Conclusions}

The versatility of isorisk methodology allows its use not only for analysing industrial risk (ex.: fire and machinery breakdown) but also for very specific applications, as presented in this document, and related to loading/unloading bays.

When in-depth analysis of the risks and a risk monitoring/control tool is needed, the use of isorisk graphs (associated with data collection checklists) is a precise and flexible tool, which can be used to evaluate a variety of problems.

Even if the obtained values are relative and not absolute, the use of the same system of weights and algorithms produces comparable results. Comparable results will allow you to compare the exposure levels of various sites.

The above described quantitative-type methodology allows for both a benchmark (to compare the risk level of various locations) and an analysis per individual locations to highlight the aspects that produce the risk level. 
66 Safety and Security Engineering V

\section{References}

[1] Forte, M., Salvador, E., Risk engineering decision tools for risk manager support, $4^{\text {th }}$ International Disaster and Risk Conference IDRC Davos 2012.

[2] Marchet, G., Calabrese A., Design and managements of loading bays in the distribution sector. PoliScript.

[3] SUVA, Check list - Pedane per raccordo rampe e nicchie di carico. Code 67066.i.

[4] SUVA, Check list-Dock levellers. Code 67065.i.

[5] Taylor B., Confined spaces - Common errors and incorrect interpretations of OSHA standard.

[6] UNI EN 1398, Dock levellers - Safety requirements. 